$62^{\text {ème }}$ Congrès de la SFCO, 02008 (2014)

DOI: $10.1051 /$ sfco/20146202008

(C) Owned by the authors, published by EDP Sciences, 2014

\title{
Evaluation du dépistage des lésions précancéreuses de la cavité buccale par les étudiants en Chirurgie Dentaire auprès d'une population à risque.
}

\author{
Heuze $\mathrm{Ch}^{1}$, Bodard AG', Bourgeois $\mathrm{D}^{2}$ \\ 1 Centre de soins et de traitements dentaires, 6-8 place Deperet, 69007, Lyon, FRANCE \\ 2 Faculte d'Odontologie, Lyon 1, 9 rue guillaume Paradin, 69008, Lyon, FRANCE
}

La détection des lésions précancéreuses de la cavité buccale représente en 2014 un réel enjeu de santé publique avec 7000 nouveaux cas par an et un taux de survie à 5 ans de $50 \%$ (Ferlay et al. 2007). Sankaranarayanan et collaborateurs, lors du seul dépistage randomisé en double aveugle réalisé entre 1996 et 2004 auprès d'un échantillon de 191873 participants, objectivent l'hypothèse que le simple dépistage des lésions précancéreuses de la cavité buccale (LPCCB) par un examen visuel permettrait de réduire la mortalité mondiale par cancers buccaux de 37.000 décès par an (Jonhson et al. 2011). Cependant, l'analyse de la littérature (Sankaranarayanan et al. 2005; Enquête UFSBD-Inca 2006; Syme et al. 2001) démontre un ressenti de formation insuffisante par les professionnels de Santé que ce soit lors de la formation initiale et lors de formation continue associé paradoxalement à un désir et un besoin exprimé de formation dans ce domaine.

L'étude débutée en décembre 2012 a pour objectif d'évaluer les valeurs prédictives diagnostiques du dépistage des LPCCB réalisé par une population d'étudiants en fin de cursus universitaire (6eme année) auprès d'une population à risque du Service de Consultation et Traitements dentaires des Hospices Civils de Lyon. Les critères d'inclusion dans l'étude sont : patient de plus de 45 ans, fumeur à raison de 20 paquets/ années et/ou consommateur de plus de 2 unités d'alcool par jour. Les critères d'exclusion sont: connaissance d'une LPCCB ou d'un carcinome épidermoïde de la cavité buccale déjà existant, consultant vivant en maison médicalisée, mauvaise compréhension du français, mobilité géographique ne permettant pas le suivi à un an et/ou refus de participer à l'étude. La première consultation [Cl1] a été réalisée par les étudiants de sixième année. La seconde consultation [Cl2] a été réalisée par un chirurgien-dentiste spécialisé en cancérologie buccale (Centre Bérard - Lyon et Unité Fonctionnelle de Chirurgie) et a été associée aux examens complémentaires nécessaires (biopsie) permettant le diagnostic formel. Une troisième consultation par le chirurgien-dentiste spécialisé est prévue à 1 an au cours de l'année 2014.

Sur les 30 patients inclus, les chirurgiens-dentistes spécialistes détectent 21 LPCCB contre 16 pour les étudiants de $6^{\circ}$ année. II existe une concordance de consensus pour 24 dossiers entre chirurgiensdentistes spécialistes et étudiants, que le dépistage soit positif ou négatif. Sur ces 24 dossiers pour lesquels le consensus est atteint, 16 présentent une positivité à l'examen avec une différence de localisation entre étudiants et spécialistes dans un cas sur deux. L'étude objective aussi une différence de diagnostic entre étudiants et praticiens auprès de 13 dossiers avec une sous estimation des cas de kératoses (3 dossiers) par les étudiants. Les analyses statistiques plus avancées sont en cours.

Le dépistage précoce des LPCCB repose certainement à l'avenir sur une concertation des différents acteurs (professionnels de santé, assurance maladie, université,...) afin d'encourager une formation initiale plus uniformisée au sein des différentes universités et de promouvoir la formation continue des chirurgiens-dentistes. Une médiatisation de ces pathologies auprès du grand public est aussi essentielle pour réduire la mortalité et la morbidité des cancers buccaux.

HEUZE Charlotte carlottaheuze@hotmail.com

This is an Open Access article distributed under the terms of the Creative Commons Attribution License 4.0, which permits unrestricted use, distribution, and reproduction in any medium, provided the original work is properly cited. 